\title{
CAD System for the Assistance of Comparative Reading for Lung Cancer Using Serial Helical CT Images
}

\author{
M. Kubo ${ }^{1}$, T. Yamamoto ${ }^{1}$, Y. Kawata ${ }^{1}$, N. Niki ${ }^{1}$, K. Eguchi ${ }^{2}$, H. Ohmatsu ${ }^{3}$, \\ R. Kakinuma ${ }^{3}$, M. Kaneko ${ }^{4}$, M. Kusumoto ${ }^{4}$, N. Moriyama ${ }^{4}$, K. Mori ${ }^{5}$, and \\ H. Nishiyama ${ }^{6}$ \\ ${ }^{1}$ University of Tokushima, Tokushima, Japan, \\ ${ }^{2}$ National Shikoku Cancer Center Hospital, Ehime, Japan, \\ ${ }^{3}$ National Cancer Center Hospital East, Chiba, Japan, \\ ${ }^{4}$ National Cancer Center Hospital, Tokyo, Japan, \\ ${ }^{5}$ National Tochigi Cancer Center Hospital, Tochigi, Japan, \\ ${ }^{6}$ Social Health Insurance Medical Center, Tokyo, Japan
}

\begin{abstract}
The objective of this study is to develop a computer-aided diagnosis (CAD) system to support comparative reading of sequential helical CT images for lung cancer screening without using film displays. The placement of pulmonary shadows between sequential helical CT images sometimes differs due to the changes in lung size and shape caused by inspiration. The proposed algorithm consists of two sections; identification of region of interest and the comparison of sequential CT images. We validated the effectiveness of the algorithm by its application to images from 60 subjects. The algorithm could compare the slice images correctly with respect to a physician's point of view. The experimental results indicate that the proposed algorithm is useful in increasing the efficiency of the mass screening process.
\end{abstract}

\section{Introduction}

Early detection and treatment is necessary to improve the recovery rate of patient with lung cancer. During mass screening, helical CT images are obtained under the following measurement conditions: $10.0 \mathrm{~mm}$ beam width; $50 \mathrm{~mA}$ tube current; and 10.0 -mm reconstructed intervals. The mass screening generates such a considerable number of images that are time-consuming to assess, it can be difficult for clinicians to make use of them in clinical setting. In particular, comparative reading of sequential helical CT images burden to be registration in lung size and shape caused by inspiration. In this paper, we analyze the motion of the pulmonary structure using the serial images taken at early diagnosis that differ with regard to the extent of inspiration. We then present a new algorithm that matches the slice position of each image in the scan sequence based on the motion of the lung. 


\section{Algorithm for Comparison of Each Slice of Serial Cases}

Expert physicians make the comparative reading of the lung based on the position of the pulmonary blood vessels in each image. We analyzed the motion of the pulmonary structure using 17 pairs of sequential images. The comparison results between each sequential image were confirmed the motion of the superior surface of diaphragm and the lung base, while the apexes of the lung and the aortic arch essentially did not moved.

We present an algorithm for each part; the upper lung and lower lung. This algorithm consists of two processes, the region detection process and the comparison process between each slice of serial cases.

In the region detection algorithm, the left and right lung regions are automatically identified in the CT image using the lung extraction method of a conventional CAD system [1]. In the comparison process, the current case is used us the reference and the retrospective case is used as the candidate. An image is selected, which is similar to the present case. This process consists of five stages as follows;

(Stage-1) Corrections of coarse difference in the scan positions using the position of the apex of lung and the backbone as reference points

(Stage-2) Matching of each image of the upper lung using lung shape [2]

(Stage-3) Matching of each sequential image of the lower lung using the pattern of the pulmonary blood vessels

(Stage-4) Evaluation of continuity between upper and lower lung results

(Stage-5) Amendment of discontinuities between results

\section{Experiment}

The proposed algorithm was tested using 3,502 images (136 pairs) from 60 subjects. The same data was visually analyzed to provide ground truth data. Table 1 shows the results of the tests, where judgment I defines the difference between ground truth and result of test is not any, judgment II defines the difference is one slice, judgment III defines the difference is two slices, and judgment IV defines the difference is more than three slices. Cumulative rate within one slice difference is 3,481 images and $99.4 \%$ in all of judgment I and II, and one within two slice difference is 3,490 images and $99.7 \%$ in all of judgment I through III.

The cases of judgment IV show three characteristics such as the shrunk lung, the expansion heart and the whitish lung's background. The proposed method using the lung shape and heart shape is difficult to apply in case of no enough inspired air.

\section{Conclusion}

We present a new CAD system to support effectually the comparative reading using the serial helical CT images for lung cancer screening without using the film display. Cumulative rate within one slice difference was 3,481 images and $99.4 \%$ in all of judgment I and II. The experimental results of the proposed algorithm indicate that 
our CAD system without using the film display is useful to increase the efficiency of the mass screening process. In future work, we will examine the proposed algorithm using many cases.

Table 1. Result of the proposed method

\begin{tabular}{|c|c|c|c|}
\hline judgment & $\begin{array}{c}\text { slice } \\
\text { difference }\end{array}$ & $\begin{array}{c}\text { No. of } \\
\text { slices }\end{array}$ & rate (\%) \\
\hline I & $0.0-0.5$ & 3227 & 92.1 \\
\hline II & $0.5-1.0$ & 254 & 7.3 \\
\hline III & $1.0-2.0$ & 9 & 0.3 \\
\hline IV & $2.0-$ & 12 & 0.3 \\
\hline
\end{tabular}

\section{References}

1. K. Kanazawa, Y. Kawata, N. Niki, et al., "Computer-aided diagnosis for pulmonary nodules based on helical CT images," Comput. Med. Imag. Graph., 22, pp.157-167, 1998.

2. Y. Ukai, N. Niki, H. Satoh, K. Eguchi et al., "Computer aided diagnosis system for lung cancer based on retrospective helical CT image," in Image Processing, Kenneth M, Hanson, ed, Proc. SPIE 3979, pp. 1028-1039, 2000. 